\title{
SIGNO DE LA “LENGUA BRILLANTE” EN ESCLEROSIS LATERAL AMIOTRÓFICA
}

\author{
VARGAS OSORIO JULIANA ${ }^{1^{*}}$, NIEBLES POLO CARLOS ${ }^{2}$ \\ ${ }^{1^{*}}$ Residente Neurología. Universidad Militar Nueva Granada, Bogotá. Hospital Militar Central. Bogotá, Colombia \\ ${ }^{2}$ Neurólogo Clínico, Docente en Neurología, Universidad Militar Nueva Granada, Bogotá. Hospital Militar Central. \\ Bogotá. Colombia \\ Correspondencia: \\ Dirección Postal: Transversal 3 \# 49-00 Facultad de Medicina. Bogotá, Colombia
}

Recibido: 28 Mayo 2014 Aceptado: 15 Febrero 2015

\begin{abstract}
Resumen
La esclerosis lateral amiotrófica (ELA) es una enfermedad neurodegenerativa. Aunque su diagnóstico sigue siendo clínico, los exámenes adicionales pueden ser utilizados para excluir otras enfermedades, así como para confirmar el diagnóstico. Uno de ellos son las neuroimagenes, donde la más utilizada es la resonancia nuclear magnética (RNM). En la literatura hay pocos signos específicos; el tracto corticoespinal, giro precentral y el cuerpo calloso son los principales sitios de alteración. Un signo patognomónico es el signo de la lengua brillante, debido a la alteración de la lengua en su estructura interna con áreas de aumento y disminución de intensidad de señal, en fases de severo compromiso bulbar con mayor intensidad en T1.
\end{abstract}

Palabras clave: signo, neurología, lengua brillante, esclerosis lateral amiotrófica, resonancia magnética nuclear, diagnóstico.

\section{“BRIGHT TONGUE” SIGN IN AMYOTROPHIC LATERAL SCLEROSIS}

\begin{abstract}
Amyotrophic lateral sclerosis is a progressive neurodegenerative disease, but its diagnosis remains clinical, additional tests may be used to exclude other diseases and to confirm the diagnosis. One of them is the images studies, and the most commonly used is magnetic resonance imaging. In literature there are few signs specific finding as major sites of alteration: the corticospinal tract, precentral gyrus, corpus callosum.. A pathognomonic sign"bright tongue sign", because alteration in the internal structure with areas of increased and decreased signal intensity in severe bulbar commitment phases with greater intensity in T1.
\end{abstract}

Keyswords: sign, neurology, brilliant tongue, amyotrophic lateral sclerosis, nuclear magnetic resonance, diagnosis. 


\title{
SINAL DA “LINGUA LUSTROSA" EM ESCLEROSE LATERAL AMIOTRÓFICA
}

\begin{abstract}
Resumo
A esclerose lateral amiotrófica é uma doença neuro-degenerativa. Embora seu diagnóstico ainda seja clínico, os examines podem-se aplicar para excluir outras doenças, assim como para confirmar o diagnóstico. Um de eles são as neuroimagens, onde as mais aplicada é a ressonância nuclear magnética (RNM). Na literatura ha poucas sinales específicas: o trato corticoespinal, giro precentrarl y o corpo caloso são os principais sites de alteração. Una sinal patognómica é a sinal da língua lustrosa, devido a alteração da língua na estrutura interna com áreas de aumento e diminuição de intensidade da sinal, em fases de severo compromisso bulbar com maior intensidades em T1.
\end{abstract}

Palavras-chave: sinal, neurologia, língua lustrosa, esclerose lateral amiotrófica, ressonância magnética nuclear, diagnóstico.

\section{Introducción}

La esclerosis lateral amiotrófica (ELA) es una enfermedad neurodegenerativa progresiva y fatal que afecta la función motora y el sistema extramotor. Estructuralmente afecta componentes del sistema motor como el tracto corticoespinal, la motoneurona inferior afecta el tallo cerebral y/o el cuerno anterior de la médula, el cuerpo calloso también ha mostrado estar involucrado en algunos casos de ELA (1).

Se solicita de rutina RNM de cerebro y de columna vertebral como parte del diagnóstico diferencial (2), actualmente se han desarrollado aplicaciones avanzadas para visualizar mejor las características propias de la enfermedad; un ejemplo característico de diagnóstico diferencial es degeneración de la columna cervical que puede resultar en la combinación de lesiones clínicas de motoneurona superior por mielopatía cervical y de motoneurona inferior por radiculopatía. En la literatura hay pocos signos de IRM reportados que puedan soportar el diagnóstico directamente $(, 3,4,5)$. En este contexto hiperintensidades del tracto corticoespinal (corteza precentral, corona radiada, brazo posterior de capsula interna, pedúnculo cerebrales, puente) que representan degeneración de este tracto (6) o algunas veces en la médula también han sido descritas; además atrofia del giro precentral, con baja sensibilidad, o hipointensidades en el giro precentral "línea oscura motora" en T2, lesiones hiperintensas en el cuerpo calloso representando degeneración de las fibras comisurales que interconectan las corteza motoras bilaterales (7).

En la década pasada se describieron signos patognomónicos de esta enfermedad dentro de los cuales se encuentra el signo de la lengua brillante; en 1989 Gwenaelle y Cha, realizaron un estudio de casos y controles donde compararon la lengua de 16 personas con esclerosis lateral amiotrófica $(8,9)$, con 20 pacientes controles, por medio de RNM, encontrando que la lengua de los pacientes con ELA presentaba anormalidades en la forma, tamaño, posición y estructura interna, el tamaño fue menor de dos tercios que lo normal; la forma fue más rectangular y cuadrada en lugar de ser curveada como la normal, la posición dependió del estadio de la enfermedad, encontrando en los estadios avanzados mayor debilidad en los músculos por lo que no estaba en contacto con el paladar duro o blando; las bandas radiales normales de la lengua a menudo estaban ausentes en la ELA al igual que las dos bandas curvo líneas que corren paralelas a las bandas radiales, finalmente encontraron alteración en la estructura interna con áreas de aumento y disminución de intensidad de señal, un estudio posterior de Konagaya y colaboradores (10) reportado en 1990, evaluó hallazgos en la lengua de pacientes con enfermedades neurodegenerativas como esclerosis lateral amiotrófica, atrofia muscular bulboespinal, Síndrome de ShyDrager, encontraron en Esclerosis lateral amiotrófica con afección bulbar severa: atrofia, irregularidad en lengua con incremento de la señal en T1 IRM; casos más leves de afección bulbar mostraron compromiso mixto de áreas de hiperintensidad y normointensidad en lengua; casos de Esclerosis sin compromiso bulbar, se evidenció incremento de la intensidad, sugiriendo compromiso subclínico del músculo lingual. El caso de atrofia muscular bulboespinal, también mostro atrofia en lengua con incremento difuso de intensidad en IRM. El caso de Shy Drager mostró forma normal de la lengua, sin incremento de la intensidad, pero con hipotonía. Concluyendo finalmente que la lengua en RNM es útil su evaluación en síntomas bulbares. 


\section{Descripción del paciente}

Paciente femenina de 56 años con cuadro clínico progresivo de 6 meses de evolución de disartria de lenta progresión, disfagia para sólidos, pérdida de peso cuantificada en $8 \mathrm{Kg}$, pérdida de fuerza en región distal de manos bilateral para realizar acciones finas; el examen neurológico mostró fasciculaciones en tres segmentos (bulbar, torácica, lumbar) asociado a hiperreflexia en miembros inferiores. Estudios neurofisiológicos iniciales reportan polineuropatía sensitivo motora axonal con leve componente mielínico de 4 extremidades de evolución crónica, por incongruencia clínica con el informe se repitió el estudio a los 3 meses, encontrando neuroconducciones normales y una electromiografía que reveló denervación y reinervación bulbar, torácica y lumbar, características sugestivas de enfermedad de motoneurona definitiva por compromiso de tres segmentos, la RNM cerebral muestra en la secuencia T1 imagen hiperintensa en lengua (Figura 1.), consistente con denervación crónica de la musculatura de la lengua por tejido graso, apoyando el diagnóstico de Esclerosis lateral amiotrófica (11).

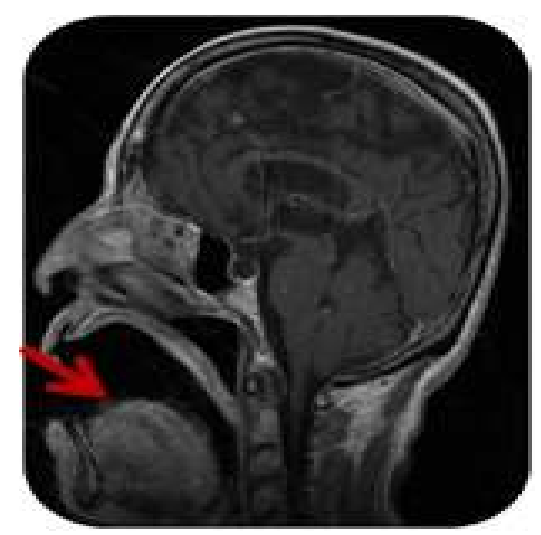

Figura 1. Paciente del caso

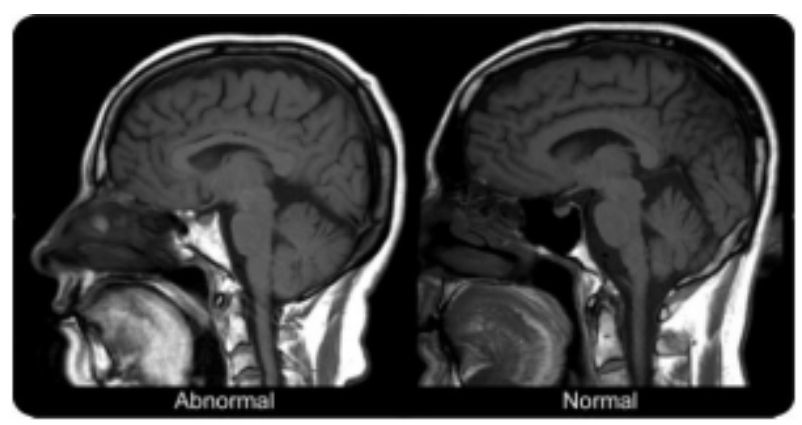

Figura 2. Tomado de Neurology 2011(12).

\section{Discusión}

La esclerosis lateral amiotrófica o enfermedad de motoneurona (o enfermedad de Lou Gehrig), es un desorden neurodegenerativo crónico, causada por degeneración de la primera motoneurona en la corteza cerebral, afectando más severamente las neuronas del giro precentral que el poscentral, este desorden lleva a una degeneración Walleriana del tracto corticoespinal y corticobulbar, que parte de la corteza motora, pasa por cápsula interna, pedúnculos cerebrales, puente y cordón espinal; hay también compromiso de la segunda neurona en el asta anterior, estos sitios se muestran comprometidos en neuroimagenes $(13,14)$.

Los criterios el Escorial usados para el diagnóstico de Esclerosis Lateral Amiotrófica usan resonancia cerebral simple para excluir otras enfermedades que simulan la ELA, técnicas de RNM convencionales no están disponibles para demostrar degeneración del tracto cortico espinal (15).

En la actualidad hay incremento en técnicas de neuroimagenes avanzadas tales como resonancia con espectroscopia, difusor tensor, imágenes de resonancia funcional, las cuales muestran signos indirectos de neurodegeneración del tracto corticoespinal, de acuerdo a los estudios la RNM con espectroscopia y tensor difusión tienen alta sensibilidad y especificidad incluso en los estadios tempranos de la enfermedad $(13,14,16,17)$.

Sin embargo en muchas instituciones de países subdesarrollados no tienen disponibles estas imágenes avanzadas por lo que signos patognomónicos como el signo de la lengua brillante en secuencias T1 de resonancia nuclear magnética; tal como el observado en nuestra paciente, lo que apoyo el diagnóstico junto con la clínica y los estudios neurofisiológicos. Este signo se encuentra en pocos casos reportados en la literatura reportados desde 1989 (9) en un estudio de casos y controles reportado por Cha y colaboradores, el cual describió las características de la lengua en pacientes con ELA de manera clínica y radiológica y posteriormente en 1990 por Konagaya y colaboradores (10) que estudiaron hallazgos en lengua en enfermedades neurodegenerativas como esclerosis lateral amiotrófica, atrofia muscular bulbo espinal, Síndrome de ShyDrager, encontraron que a pesar que no es un signo específico para Esclerosis Lateral amiotrófica es un buen elemento que ayuda a apoyar el diagnótico.

\section{Conflicto de intereses}

Los autores declaramos no tener de manera directa o indirecta, ningún tipo de conflicto de intereses financieros, académicos o laborales que puedan poner en peligro la validez de este estudio. 


\section{Financiación}

Este trabajo se realizó con el apoyo financiero de los investigadores.

\section{Referencias}

1. Turner R, Agosta F, Bede P. Neuroimaging in amyotrophic lateral sclerosis. Biomarkers Me. 2012;6(3): 319-337.

2. Kassubek J, Ludolph A, Müller H. Neuroimaging of motor neuron diseases. Ther Adv Neurol Disord. 2012; 5(2): 119-127.

3. Bede P, Hardiman O. Lessons of ALS imaging: Pitfalls and future directions. A critical review. Neuroimage: Clinical. 2014:436-443.

4. Toro J, Reyes S, Useche N. Neuroimaging in amyotrophic lateral sclerosis. BMJ Case Rep. 2013

5. Foerster BR1, Welsh RC, Feldman EL. 25 years of neuroimaging in amyotrophic lateral sclerosis. Nat Rev Neurol. 2013; 9(9):51324.

6. Goodin DS, Rowley HA, Olney RK. Magnetic resonance imaging in amyotrophic lateral sclerosis. Ann Neurol. 1988; 23(4):418420 .

7. Rinsho Shinkeigaku. MR imaging of the brain of amyotrophic lateral sclerosis. Department of Neuroradiology. 2002 (11):1139-1140.

8. Douaud G, Filippini N. Integration of structural and functional magnetic resonance imaging in amyotrophic lateral sclerosis. Brain 2011;(134):3470-3479.

9. Cha $\mathrm{CH}$, Patten BM. Amyotrophic lateral sclerosis: abnormalities of the tongue on magnetic resonance imaging. Ann Neurol 1989;(25):468-472.

10. Konagaya M, Konagaya $\mathrm{Y}$, Konishi $\mathrm{T}$, et al. MRI findings of the tongue in neurodegenerative diseases with bulbar sign. 1990;(30):665-667.

11. Michael D. Fox and Adam B. Cohen. "Bright tongue sign" in ALS. Neurology.2012;(79);1520.

12. Provenzale J. Dementias and degenerative disorders. In: Osborn AG, Blaser SI, Salzman KL, editors. Diagnostic Imaging. Brain. 1st ed. Salt Lake City: Amirsys; 2004: 86-89.

13. Berciano J, Zarranz JJ, Polo JM. Enfermedad degenerativa espinal y espinocerebelosas. In: Zarranz JJ, editors. Neurología. 3rd ed. Madrid: Elsevier España; 2003. 689-95.

14. Da Rocha AJ, Oliveira AS, Fonseca RB, Maia et al. Detection of corticospinal tract compromise in amyotrophic lateral sclerosis with brain MR imaging: relevance of the T1-weighted spin-echo magnetization transfer contrast sequence. AJNR Am J Neuroradiol. 2004; (25):1509-1515.

15. Brooks BR, Miller RG, Swash M, Munsat TL. El Escorial revisited: revised criteria for the diagnosis of amyotrophic lateral sclerosis Amyotroph Lateral Scler Other Motor NeuronDisord. 2000: 293-299.
16. Comi G, RovarisM, Leocani L. Neuroimaging in amyotrophic lateral sclerosis. Eur J Neurol. 1999;(6):629-637.

17. Filippi M, Agosta F, Abrahams S, Fazekas F, et al. EFNS guidelines on the use of neuroimaging in the management of motor neuron diseases. Eur J Neurol. 2010;(17):526-33. 